\title{
Supervivencia de pacientes con metástasis cervical de primario desconocido en el Instituto Nacional de Oncología y Radiobiología. 2007-2012
}

\section{Survival of patients with cervical metastasis of unknown primary at the National Institute of Oncology and Radiobiology. 2007-2012}

\author{
Zeila Omar R. ${ }^{1}$, Pedro Pablo Morales H. ${ }^{1}$, Maykel Pérez V. ${ }^{1}$, Carlos Javier F. ${ }^{1}$, \\ Roberto Ortiz B. ${ }^{1}$ Johannes René Mestre C. ${ }^{1}$
}

'Servicio de Cirugía de Cabeza y Cuello. Instituto Nacional de Oncología y Radiobiología.

Calle 29 esquina F. Vedado.

La Habana, Cuba.

Los autores declaran no tener conflictos de interés.

Recibido el 10 de abril de 2020. Aceptado el 13 de mayo de 2020

Correspondencia: Zeila Omar R

Calle 12 Edificio 9 apto. 4 Versalles. CP:90700

Santiago de Cuba, Cuba Email: omarzeila99@gmail.com

\section{Resumen}

Introducción: Las metástasis cervicales con primario oculto representan el 5\% de los tumores de cabeza y cuello. Su diagnóstico y tratamiento han sufrido modificaciones con los años según las diferentes investigaciones, con vistas a mayores tasas de supervivencia. Objetivo: Determinar la supervivencia global y según diferentes variables. Material y Método: Investigación retrospectiva, descriptiva y longitudinal de 96 pacientes diagnosticados en el Instituto Nacional de Oncología y Radiobiología (INOR) en el periodo 2007-2012, manteniéndose el seguimiento hasta el 31 de diciembre de 2017. Resultados: De los pacientes estudiados, 65 eran hombres $(67,7 \%)$ y 31 mujeres $(32,3 \%)$, para una razón de 2,1:1, con una media de edad de 61,4 años. El tabaquismo estuvo presente en el 64,6\% de la población. El nivel ganglionar más afectado fue el II y 41 pacientes $(42,7 \%)$ presentaron una enfermedad N1 al diagnóstico. En 42 pacientes $(43,8 \%)$ estuvo presente el grado histológico poco diferenciado. Al 70,9\% se les practicó cirugía con adyuvancia o sin ella y el $86,5 \%$ tuvo respuesta completa. El tumor primario apareció en el 13,5\% de los casos, más frecuente en amígdala. La supervivencia global a los 5 años fue del $74 \%$ y resultaron variables determinantes de supervivencia el estado ganglionar y la extensión extraganglionar. Los resultados coinciden con la literatura revisada. Conclusión: La supervivencia global a los 5 años de los pacientes con metástasis cervical de primario oculto es elevada, tiene tendencia a mejorar con el paso de los años y está determinada por el estado ganglionar y la extensión extracapsular de forma inversamente proporcional.

Palabras clave: primario desconocido, metástasis cervical, cáncer.

\section{Abstract}

Introduction: Cervical metastases with occult primary represent $5 \%$ of head and neck tumors. Its diagnosis and treatment have been modified over the years according to different investigations, with a view to higher survival rates. Aim: To determine the overall survival and according to different variables. Material and Method: A retrospective, descriptive and longitudinal investigation of 96 patients diagnosed at INOR in the period 2007-2012 was carried out, with follow-up until December 31, 2017. Results: Of the patients studied, 65 were men (67.7\%) and 31 women (32.3\%), for a ratio of 2.1: 1, with a mean age of 61.4 years. Smoking was present in the $64.6 \%$ of the population. The most affected lymph node level was level II and 42 patients (43.8\%) presented N1 disease at diagnosis. Poorly differentiated histological grade was present in 42 patients (43.8\%). $70.9 \%$ underwent surgery with or without adjuvant surgery and $86.5 \%$ had a complete response. The primary tumor appeared in 13.5\% of cases, more frequent in the amygdala. Overall survival at 5 years was $74 \%$, and survival variables were lymph node status and extranodal extension. The results coincide with the reviewed literature. Conclusion: The overall 5-year survival of patients with occult primary cervical metastasis is high, tends to improve over the years, and is determined by lymph node status and extracapsular extension in an inversely proportional manner.

Keywords: unknown primary, cervical metastasis, cancer. 


\section{Introducción}

El cáncer de cabeza y cuello es uno de los principales problemas de salud de estos tiempos. En 2018 se diagnosticaron 887.659 nuevos casos de tumores malignos de cavidad oral, laringe, faringe y glándulas salivales, en todo el mundo, mientras que murieron 453.307 personas por esta causa $^{1,2}$. Desde 1990 se recogen publicaciones de estudios donde se usa la técnica de reacción en cadena de la polimerasa (PCR por sus siglas en inglés) para identificar ADN de HPV (Human Papilomavirus) en carcinomas escamosos de cabeza y cuello y su distribución genotípica, con el objetivo de dilucidar la carcinogénesis mediada por el virus. De ahí surge el carcinoma escamoso de cabeza y cuello asociado a HPV como una entidad más frecuente en la orofaringe, que afecta a individuos jóvenes y saludables con poca o ninguna exposición al tabaco, y que se caracteriza por alta respuesta al tratamiento y buen pronóstico, además de tumores primarios muy pequeños u ocultos ${ }^{3-6}$.

Entre un 2\% y 5\% de los casos se confirma histológicamente la metástasis ganglionar cervical y el tumor primario no se encuentra luego de agotar todos los procederes diagnósticos, es lo que se conoce como primario oculto o desconocido, para varios autores considerado un síndrome. Esto ha motivado un sinnúmero de investigaciones con el fin de perfeccionar las vías para encontrar el tumor primario, así como para definir qué tratamiento es el más idóneo. El diagnóstico y manejo de las metástasis cervicales de carcinoma escamoso con primario desconocido ha sido un tema controversial en los últimos años donde el consenso sobre el uso de tomografía computarizada con tomografía por emisión de positrones (PET-CT) y la realización de amigdalectomías era defendido por algunos investigadores y cuestionado por otros, así como cuál era la modalidad de tratamiento que garantizaría mayores tasas de sobrevida y de intervalo libre de enfermedad. Los últimos hallazgos sobre la influencia de la infección por HPV como mecanismo y etiología de carcinoma escamoso con diferente comportamiento y mejor pronóstico, ha revolucionado las estrategias de diagnóstico y tratamiento para el cáncer de cabeza y cuello, incluyendo las metástasis con primario oculto ${ }^{7}$.

\section{Objetivo}

Determinar la supervivencia de los pacientes con metástasis cervical de carcinoma escamoso de origen desconocido en el Instituto Nacional de Oncología y Radiobiología, en el período comprendido entre el 1 de enero de 2007 y el 31 de diciembre de 2012. Caracterizar a los pacientes con metástasis cervical de carcinoma escamoso de origen desconocido, según aspectos epidemiológicos y clínico-anatomopatológicos. Determinar la supervivencia de los pacientes según diferentes variables.

\section{Material y Método}

\section{Aspectos generales del estudio}

Se realizó un estudio observacional, analítico, longitudinal y retrospectivo de los pacientes con metástasis cervical de carcinoma escamoso de origen desconocido inscritos en el INOR en el período comprendido entre el 1 de enero de 2007 y el 31 de diciembre de 2012. El tiempo de seguimiento fue hasta el 31 de diciembre de 2017.

\section{Definición del universo de estudio y muestra}

El universo de estudio estuvo constituido por todos los pacientes con metástasis cervical de carcinoma de origen desconocido, inscritos en el INOR. De ellos se seleccionó una muestra constituida por los 96 pacientes inscritos en el período comprendido entre enero de 2007 y diciembre de 2012, según los criterios de inclusión.

\section{Criterios de inclusión}

Diagnóstico histológico de metástasis de carcinoma escamoso. Que se encuentren incorporados a las consultas de seguimiento del INOR. Pacientes en los cuales no apareció el tumor primario antes del tratamiento.

Se tuvieron en cuenta los siguientes conceptos esenciales en el análisis de supervivencia:

Fecha inicial: fecha del diagnóstico.

Fecha de última noticia: fecha correspondiente a la última información que se tiene del caso.

Seguimiento: es la observación de los individuos a partir de la fecha inicial, para conocer su estado vital (vivo, fallecido o desconocido). 
Período de seguimiento: es el tiempo transcurrido entre la fecha de inicio $(01 / 01 / 07)$ y la fecha de corte del estudio (31/12/17).

Fecha de corte del estudio: fecha fijada por el investigador para el término del seguimiento de los pacientes $(31 / 12 / 17)$.

Tiempo de supervivencia: es el intervalo de tiempo transcurrido entre la fecha de inicio y la fecha de última noticia.

\section{Técnicas y procedimientos}

\section{Obtención y recolección de la información}

Para lograr el mayor grado de validez científica posible basada en un marco teórico bien fundamentado, se realizó una revisión bibliográfica previa a la confección del proyecto $y$, posteriormente, durante todo el proceso de ejecución de la investigación, del tema en general y de las temáticas particulares, en la biblioteca del INOR y en las bases de datos LILACS, MEDLINE, SCIELO, sitios web como el de la OPS, INFOMED y NCI. Se realizaron además consultas de asesoramiento con especialistas de experiencia relacionados con el tema estudiado.

Los datos primarios se obtuvieron mediante la revisión de las historias clínicas de los pacientes incluidos en el estudio. Para la identificación de los casos con metástasis cervical de origen desconocido se revisó la base de datos de reporte de cáncer con ese diagnóstico del departamento de registros médicos del INOR, detectándose además del diagnóstico de carcinoma escamoso, adenocarcinoma, melanoma y otros. En total se exploraron 122 historias clínicas, de las cuales 6 correspondieron a diagnóstico de linfoma y en otras 20, inscritas como metástasis sin primario conocido, apareció el primario en los estudios de extensión antes del tratamiento, por lo que no se incluyeron en el estudio. Al final se recogieron los datos de 96 historias clínicas. Los datos personales, epidemiológicos y los relacionados con el tratamiento de los casos seleccionados se recolectaron en una hoja de recogida de información. Se construyó una base de datos, utilizando el programa Microsoft Excel 2010, donde se sintetizó la información recabada de las historias clínicas.

\section{Procesamiento de la información}

Los datos recopilados en la base de datos se introdujeron y procesaron en el programa estadístico SPSS, en versión 20.0, con un ordenador con procesador Core i3. Se emplearon como medidas de resumen de los mismos el porcentaje, la media aritmética y la desviación estándar. Los resultados obtenidos se mostraron en tablas y gráficos para facilitar su comprensión y análisis.

El tratamiento estadístico de los datos se detalla a continuación: obtención de las distribuciones de frecuencias. La información correspondiente a variables cualitativas se resumió mediante frecuencias absolutas y relativas y para las variables cuantitativas se utilizó la media y la desviación estándar. Se hizo estimaciones puntuales y por intervalos, con nivel de confiabilidad del 95\%, de media y de proporciones, considerando como sus estimadores las medidas de resumen utilizadas. Las estimaciones de los tiempos de supervivencia se realizó a través del método de Kaplan-Meier y regresión mediante los modelos de Cox. Se realizaron el análisis de forma global y también en función de los diversos factores pronósticos que pueden afectar la supervivencia. Para probar si las características de interés están asociadas se aplicó la prueba de Chi cuadrado o la prueba t para muestras independientes, en correspondencia al tipo de variable. Para las pruebas de hipótesis se consideró un nivel de significación estadística de 0,05.

\section{Análisis y sintesis de los datos}

El método de análisis y síntesis se aplicó para el estudio detallado de la información procesada con el objetivo de establecer nexos entre las variables y caracterizar la entidad estudiada. Los resultados fueron descritos y comentados de acuerdo con la literatura revisada, las sugerencias, el apoyo de tutores y asesores y la experiencia acumulada por el autor durante su formación. Finalmente, mediante los procesos inductivos y deductivos se pudo arribar a conclusiones y recomendaciones.

\section{Resultados}

La mayor cantidad de pacientes se diagnosticó en el año 2009, con 22 pacientes, para un $22,9 \%$ (Tabla 1). El rango de edad estuvo 
entre los 30 y los 96 años, siendo la media de 61,4 años con una desviación estándar de 11,89 años. Dentro de los grupos de edad el más numeroso fue el de 60 a 69 años con 36 pacientes $(37,5 \%)$, y el de menos frecuencia el grupo de menores de 40 años con solo 4 frecuencias $(4,2 \%)$. De los pacientes estudiados, 65 eran hombres $(67,7 \%)$ y 31 eran mujeres $(32,3 \%)$, para una razón de 2,1:1.

El 70,8\% de los pacientes presentó la metástasis en el nivel II (68 pacientes), en contraste solo 1 paciente tuvo afectación del nivel I (1,0\%) (Tabla 2). En cuanto al estado ganglionar según el TNM, el 42,7\% de los pacientes $(\mathrm{N}=41)$ se etapificó $\mathrm{N} 1$, siendo menos frecuente el estadio N2c con solo 2 pacientes $(2,1 \%)$. La extensión extraganglionar estuvo presente en 26 pacientes para un 27,1\%, y el grado de diferenciación más frecuente fue el poco diferenciado (42 pacientes para un $43,8 \%)$

De los 26 pacientes que presentaron extensión extraganglionar $13(50,0 \%)$ eran N3 (Tabla 3). El análisis de contingencia entre ambas variables mostró una significación estadística $\mathrm{p}=0,002$, lo cual evidencia una estrecha relación de dependencia entre ambas. No obstante, llama la atención que a pesar de ser N1, 4 pacientes $(15,2 \%)$ presentaron extensión extraganglionar.

En cuanto a la evaluación de la respuesta, 83 pacientes $(86,5 \%)$ fueron evaluados como respuesta completa. Los 3 pacientes no evaluados fueron aquellos que no recibieron tratamiento (Tabla 4).

El tratamiento que más se aplicó fue la cirugía seguida de radioterapia en 47 pacientes $(49,0 \%)$. Al sumar los porcentajes de todos los pacientes en los que se usó la cirugía ya sea sola o con adyuvancia se obtuvo un $70,9 \%$. Tres pacientes no fueron tributarios de ningún tratamiento oncoespecífico. Hubo 6 pacientes tributarios de cirugía, pero no la recibieron por criterios de inoperabilidad como negativa del paciente y riesgo quirúrgico elevado. El 87\% (36 pacientes) de los N1 fueron tratados con cirugía sola y cirugía con radioterapia adyuvante. Los pacientes que recibieron quimioterapia adyuvante tenían factores de mal pronóstico en la biopsia postquirúrgica. La cirugía sin adyuvancia solo se usó en $\mathrm{N} 1$ y un N2A (Tabla 5).

\begin{tabular}{|c|c|c|c|}
\hline Variable & & Frecuencia & $\%$ \\
\hline \multirow[t]{6}{*}{ Año de diagnóstico } & 2007 & 9 & 9,4 \\
\hline & 2008 & 14 & 14,6 \\
\hline & 2009 & 22 & 22,9 \\
\hline & 2010 & 19 & 19,8 \\
\hline & 2011 & 16 & 16,7 \\
\hline & 2012 & 16 & 16,7 \\
\hline \multirow[t]{6}{*}{ Grupos etarios } & $<40$ & 4 & 4,2 \\
\hline & $40-49$ & 10 & 10,4 \\
\hline & $50-59$ & 25 & 26,0 \\
\hline & $60-69$ & 36 & 37,5 \\
\hline & $70-79$ & 15 & 15,6 \\
\hline & $>79$ & 6 & 6,3 \\
\hline \multirow[t]{2}{*}{ Sexo } & Femenino & 31 & 32,3 \\
\hline & Masculino & 65 & 67,7 \\
\hline \multirow[t]{2}{*}{ Tabaco } & Sí & 62 & 64,6 \\
\hline & No & 34 & 35,4 \\
\hline \multirow[t]{2}{*}{ Alcohol } & Sí & 18 & 18,8 \\
\hline & No & 78 & 81,3 \\
\hline
\end{tabular}

Tabla 2. Características clínicas e histológicas. INOR. 2007-2012

\begin{tabular}{|c|c|c|c|}
\hline Variable & & $\begin{array}{c}\text { Fre- } \\
\text { cuencia }\end{array}$ & $\%$ \\
\hline \multirow{5}{*}{$\begin{array}{l}\text { Nivel } \\
\text { ganglionar } \\
\text { afectado }\end{array}$} & I & 1 & 1,0 \\
\hline & $\|$ & 68 & 70,8 \\
\hline & III & 19 & 19,8 \\
\hline & IV & 4 & 4,2 \\
\hline & V & 4 & 4,2 \\
\hline \multirow{5}{*}{$\begin{array}{l}\text { Estado } \\
\text { ganglionar }\end{array}$} & N1 & 41 & 42,7 \\
\hline & $\mathrm{N} 2 \mathrm{a}$ & 21 & 21,9 \\
\hline & $\mathrm{N} 2 \mathrm{~b}$ & 9 & 9,4 \\
\hline & $\mathrm{N} 2 \mathrm{C}$ & 2 & 2,1 \\
\hline & N3 & 23 & 24,0 \\
\hline \multirow{2}{*}{$\begin{array}{l}\text { Extensión } \\
\text { extraganglionar }\end{array}$} & Sí & 26 & 27,1 \\
\hline & No & 70 & 72,9 \\
\hline \multirow{3}{*}{$\begin{array}{l}\text { Grado de } \\
\text { diferenciación }\end{array}$} & Bien diferenciado & 21 & 21,9 \\
\hline & Moderadamente diferenciado & 33 & 34,4 \\
\hline & Poco diferenciado & 42 & 43,8 \\
\hline
\end{tabular}


Tabla 3. Extensión extraganglionar según estado ganglionar. INOR. 2007-2012

\begin{tabular}{|c|c|c|c|c|c|c|}
\hline \multirow{3}{*}{$\begin{array}{c}\text { Estado } \\
\text { ganglionar }\end{array}$} & \multicolumn{6}{|c|}{ Extensión extraganglionar } \\
\hline & \multicolumn{2}{|c|}{ Sí } & \multicolumn{2}{|c|}{ No } & \multicolumn{2}{|c|}{ Total } \\
\hline & $n$ & $\%$ & $n$ & $\%$ & $n$ & $\%$ \\
\hline N1 & 4 & 15,4 & 37 & 52,9 & 41 & 42,7 \\
\hline $\mathrm{N} 2 \mathrm{a}$ & 6 & 23,1 & 15 & 21,4 & 21 & 21,9 \\
\hline $\mathrm{N} 2 \mathrm{~b}$ & 2 & 7,7 & 7 & 10,0 & 9 & 9,4 \\
\hline $\mathrm{N} 2 \mathrm{C}$ & 1 & 3,8 & 1 & 1,4 & 2 & 2,1 \\
\hline N3 & 13 & 50,0 & 10 & 14,3 & 23 & 24,0 \\
\hline Total & 26 & 27,1 & 70 & 72,9 & 96 & 100 \\
\hline
\end{tabular}

Tabla 4. Respuesta al tratamiento. INOR. 2007-2012

\begin{tabular}{lcc}
\hline Respuesta & $\mathbf{n}$ & $\%$ \\
\hline Completa & 83 & 86,5 \\
\hline Estable & 4 & 4,2 \\
\hline No & 3 & 3,1 \\
\hline Progresión & 4 & 4,2 \\
\hline Parcial & 2 & 2,1 \\
\hline Total & 96 & 100,0 \\
\hline
\end{tabular}

El tumor primario apareció en 13 pacientes $(13,5 \%)$. Al sumar los porcentajes de todos los que aparecieron en cabeza y cuello se obtuvo un $84,6 \%$. Las localizaciones más frecuentes de aparición del primario fueron amígdala $(30,8 \%)$ y nasofaringe $(23,1 \%)$ (Tabla 6$)$.

La recurrencia según el estado ganglionar ocurrió en el 19,5\% de los N1. De los 32 pacientes con N2, hubo recurrencia en 10, para un $31,3 \%$, mientras que los $\mathrm{N} 3$ hubo un $73,9 \%$ de recurrencias, por lo que la recurrencia es directamente proporcional al estado ganglionar (Tabla 7).

\section{Tabla 5. Tratamiento según estado ganglionar. INOR. 2007-2012}

\begin{tabular}{|c|c|c|c|c|c|c|c|c|c|}
\hline \multirow[t]{2}{*}{ C } & & \multicolumn{6}{|c|}{ Tratamiento } & & \multirow[t]{2}{*}{ Total } \\
\hline & & CR & CRQ & NO & Q & $\mathbf{R}$ & RQ & & \\
\hline \multirow{5}{*}{$\begin{array}{l}\text { Estado } \\
\text { ganglionar }\end{array}$} & N1 & 11 & 25 & 0 & 0 & 0 & 2 & 3 & 41 \\
\hline & N2A & 1 & 13 & 3 & 0 & 0 & 4 & 0 & 21 \\
\hline & $\mathrm{N} 2 \mathrm{~B}$ & 0 & 5 & 2 & 0 & 0 & 0 & 2 & 9 \\
\hline & $\mathrm{N} 2 \mathrm{C}$ & 0 & 1 & 0 & 0 & 1 & 0 & 0 & 2 \\
\hline & N3 & 0 & 3 & 4 & 3 & 4 & 1 & 8 & 23 \\
\hline \multicolumn{2}{|l|}{ Total } & 12 & 47 & 9 & 3 & 5 & 7 & 13 & 96 \\
\hline
\end{tabular}

C: Cirugía. CR: Cirugía + radioterapia adyuvante. CRQ: Cirugía + radioterapia y quimioterapia concurrentes. NO: sin tratamiento oncoespecífico. Q: Quimioterapia sola. R: Radioterapia sola. RQ: Radioterapia y quimioterapia concurrentes. 
Tabla 6. Aparición del tumor primario según localización. INOR. 2007-2012

\begin{tabular}{lcc}
\hline Sitio & $\mathbf{n}$ & $\%$ \\
\hline Amígdala & 4 & 30,8 \\
\hline Base lengua & 2 & 15,4 \\
\hline Hipofaringe & 1 & 7,7 \\
\hline Pulmón & 2 & 15,4 \\
\hline Supraglotis & 1 & 7,7 \\
\hline Nasofaringe & 3 & 23,1 \\
\hline Total & 13 & 100,0 \\
\hline
\end{tabular}

\section{Análisis de supervivencia}

La supervivencia global media de los pacientes con metástasis cervical sin primario conocido fue de 50,3 meses, con un intervalo de confianza al $95 \%$ de 46,3 y 54,2 meses y un error típico de 2,01 meses. A lo largo del estudio fallecieron 25 pacientes $(26,0 \%)$ por causas relacionadas a su neoplasia. En lo relativo a la probabilidad acumulada de supervivencia global se obtuvo una supervivencia de la población a los 5 años de un $74,0 \%$ (Figura 1).

\section{Supervivencia a los 5 años según diferentes variables}

El estado ganglionar es un factor determinante de supervivencia $(p=0,000)$. Los pacientes con N1 tuvieron una supervivencia a los 5 años de un $87,8 \%$ y una media de 57,5 meses, mientras que los $\mathrm{N} 3$ tuvieron una supervivencia de $43,5 \%$ a los 5 años con una media de 34 meses. Mediante el análisis por el método de regresión de Cox se obtuvo igual significación estadística $(\mathrm{p}=0,000)$ (Figura 2).

En cuanto a la extensión extraganglionar, a significación estadística tanto por el método de Kaplan-Meier como regresión de Cox para esta variable fue de $p=0,000$. De los 26 pacientes con extensión extracapsular, por sus siglas en inglés ENE (extranodal extension) fallecieron 12 con una supervivencia a los 5 años del 53,8\%, mientras que de los 70 que no presentaron ENE, fallecieron 13, con una supervivencia a los 5 años del $81,4 \%$ (Figura 3). La supervivencia media de los pacientes con ENE fue de 39,4 meses y la de los que no tuvieron ENE, de 54,3 meses.

\begin{tabular}{|c|c|c|c|c|}
\hline \multirow{2}{*}{\multicolumn{2}{|c|}{$\begin{array}{l}\text { Estado } \\
\text { ganglionar }\end{array}$}} & \multicolumn{3}{|c|}{ Recurrencia } \\
\hline & & \multirow{2}{*}{$\begin{array}{l}\text { No } \\
33 \\
80,5\end{array}$} & \multirow{2}{*}{$\begin{array}{c}\text { Sí } \\
8 \\
19,5\end{array}$} & \multirow{2}{*}{$\begin{array}{c}\text { Total } \\
41 \\
100,0\end{array}$} \\
\hline N1 & $\begin{array}{l}n \\
\%\end{array}$ & & & \\
\hline N2A & $\begin{array}{l}n \\
\%\end{array}$ & $\begin{array}{l}13 \\
61,9\end{array}$ & $\begin{array}{c}8 \\
38,1\end{array}$ & $\begin{array}{c}21 \\
100,0\end{array}$ \\
\hline $\mathrm{N} 2 \mathrm{~B}$ & $\begin{array}{l}n \\
\%\end{array}$ & $\begin{array}{c}8 \\
88,9\end{array}$ & $\begin{array}{c}1 \\
11,1\end{array}$ & $\begin{array}{c}9 \\
100,0\end{array}$ \\
\hline $\mathrm{N} 2 \mathrm{C}$ & $\begin{array}{l}\mathrm{n} \\
\%\end{array}$ & $\begin{array}{c}1 \\
50,0\end{array}$ & $\begin{array}{c}1 \\
50,0\end{array}$ & $\begin{array}{c}2 \\
100,0\end{array}$ \\
\hline N3 & $\begin{array}{l}n \\
\%\end{array}$ & $\begin{array}{c}6 \\
26,1\end{array}$ & $\begin{array}{l}17 \\
73,9\end{array}$ & $\begin{array}{c}23 \\
100,0\end{array}$ \\
\hline
\end{tabular}

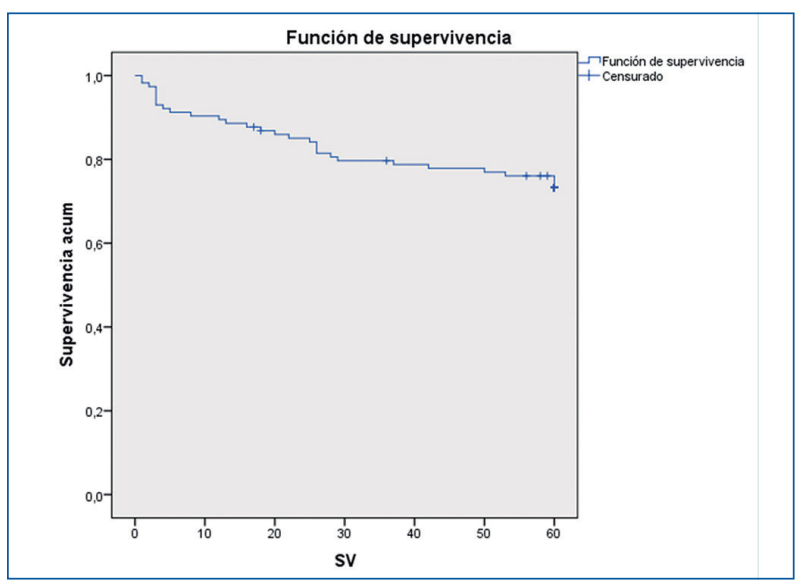

Figura 1. Probabilidad de supervivencia global acumulada en los pacientes con metástasis cervical de origen desconocido. INOR. 2007-2012.

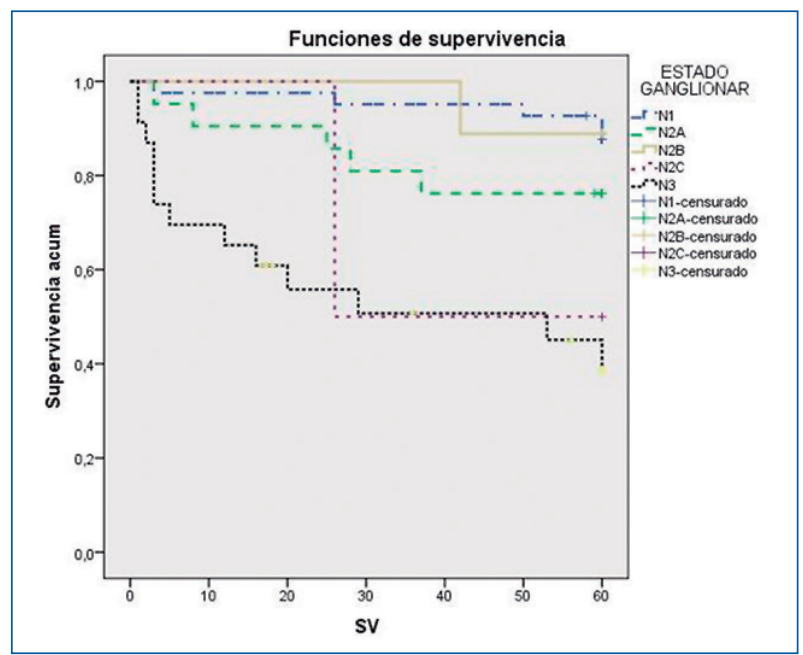

Figura 2. Probabilidad se supervivencia acumulada según estado ganglionar. INOR. 2007-2012. 


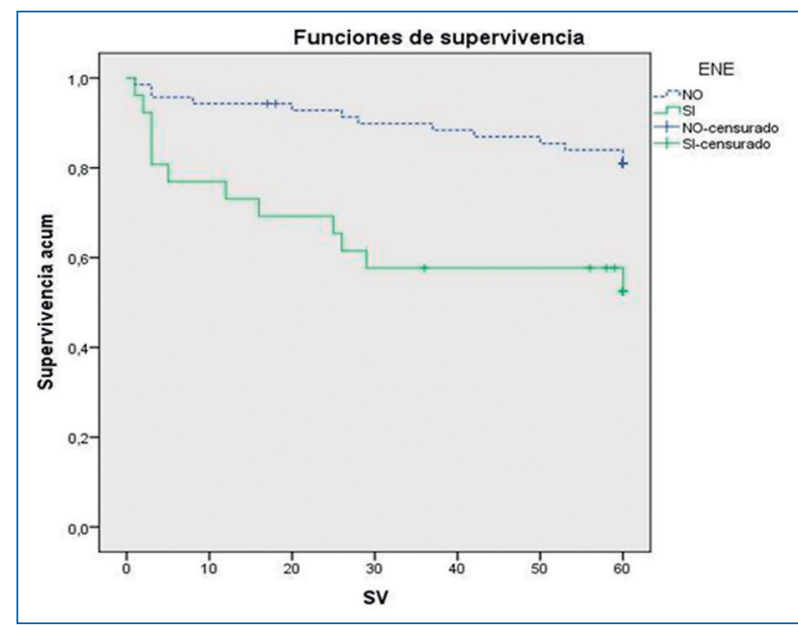

Figura 3. Probabilidad se supervivencia acumulada según extensión extraganglionar. INOR. 2007-2012.

\section{Discusión}

Las metástasis cervicales de carcinoma escamoso de origen desconocido son infrecuentes en comparación con el resto de las neoplasias malignas de cabeza y cuello, con una incidencia de entre un $2 \%$ y un $5 \%$. Desde el punto de vista epidemiológico, en nuestro estudio predominaron los hombres, en la sexta década y con práctica de hábitos tóxicos. Motz y cols. ${ }^{8}$ reportaron que el $70 \%$ de los pacientes eran fumadores, una media de edad de 57,3 años y un predominio de los hombres $(88,1 \%)$. Yildirim y cols. ${ }^{9}$ obtuvieron una media de edad de 61,5 años y Miller y cols. ${ }^{10}$, encontraron el tabaquismo en el 68\% de los casos.

En cuanto a la presentación clínica de la enfermedad generalmente afecta los niveles ganglionares superiores, y existe una tendencia al diagnóstico cada vez más temprano en cuanto al estado ganglionar. Todos los autores coinciden en que el nivel II es el más afectado seguido del nivel III y en todos los estudios predominaron los carcinomas poco diferenciados.

En cuanto al estado ganglionar en nuestra investigación encontramos un predominio de los estadios tempranos. En el estudio de Miller y cols. ${ }^{10}$ el $41,2 \%$ de los casos eran N1 y no hubo N2C. En varios estudios sobre todo aquellos que incluyen pacientes de antes del año 2000 existe un predominio de los N2. Esto puede explicarse por un mayor conocimiento de la población que acuden más temprano al especialista, así como una mayor experiencia en el manejo de estas lesiones por parte de los médicos.

El tratamiento de las metástasis ganglionares cervicales de origen desconocido ha sido controversial en cuanto si la cirugía ofrece mejores resultados que la quimiorradiación. Los últimos consensos sugieren cirugía a los tumores resecables y luego adyuvancia con quimiorradiación en dependencia del estado ganglionar y de la extensión extracapsular. También sugieren que los pacientes con N1 sin factores postquirúrgicos adversos pueden seguirse bajo observación estricta. El tipo de cirugía siempre debe abarcar los niveles del I al $V^{11,12}$. En nuestro estudio predominaron los pacientes tratados con cirugía y adyuvancia. Según el estudio de Amsbaugh y cols. ${ }^{13}$, el $56,1 \%$ de los pacientes recibieron cirugía seguida de adyuvancia ya sea con radioterapia o con quimiorradiación, mientras que al 43,9\% no se le practicó cirugía. Su principal resultado fue que el tratamiento con disección de cuello mejora la supervivencia libre de recurrencia. En el metaanálisis realizado por Xiaomei y cols. ${ }^{14}$ concluyeron que la cirugía seguida de radioterapia bilateral al cuello puede ser la opción preferida de tratamiento y se asocia a mejor control regional y supervivencia, aunque esta recomendación no está basada en ensayos aleatorizados. Según Arosio y cols. ${ }^{15}$ los pacientes con enfermedad N1 y N2A sin extensión extraganglionar pueden recibir una sola modalidad de tratamiento.

En cuanto a la aparición del tumor primario la mayoría de los autores coinciden en que generalmente se localizan en cabeza y cuello y específicamente en la orofaringe. Nieder y cols. ${ }^{16}$ obtuvieron como resultado que el primario apareció entre un 2\% y 13\% de los casos con una media de 9,5\%. Según Weber y cols. ${ }^{17}$ el tumor primario apareció en el $44 \%$ de los casos y el $76 \%$ se encontraba en cabeza y cuello. Yoo y cols. ${ }^{18}$ encontraron el primario luego del tratamiento en el 80,8\% de los casos, de ellos, el $61 \%$ en amígdala, el $18 \%$ en base de lengua, el $11,8 \%$ en nasofaringe y el $8,5 \%$ en senos piriformes. Cianchetti y cols. ${ }^{19}$ refieren que el primario fue encontrado en el $29,2 \%$ de los casos y el sitio más frecuente de aparición 
fue fosa amigdalina $(44,7 \%)$ seguido de base de lengua $(43,9 \%)$.

La recurrencia más frecuente en nuestro estudio ocurrió a nivel regional. Según el estado ganglionar cuanto más avanzada la enfermedad fue mayor la recurrencia. Esto se corresponde con lo publicado por el National Cancer Institute ${ }^{20}$ donde la supervivencia libre de enfermedad a los 3 años es de 50\% de los N1, 38\% de los N2 y 26\% de los N3. Al Kadah y cols. ${ }^{21}$ en Alemania obtuvieron que el 31\% de los pacientes tuvieron recurrencia local y el $10 \%$ metástasis a distancia, Miller y cols. ${ }^{10}$ obtuvieron como resultado un 17,6\% de recurrencias y Amsbaugh y cols. ${ }^{13}$ un $44,1 \%$.

\section{Análisis de supervivencia global}

$\mathrm{Al}$ comparar nuestro resultado con investigaciones internacionales encontramos que los autores que coinciden con nuestro estudio son Dragan y cols. ${ }^{22}$, Zhou y cols. ${ }^{23}$ y Mizuta y cols. ${ }^{24}$, con tasas de supervivencia global a los 5 años de $76 \%, 73 \%$ y $72,5 \%$ respectivamente. Huo y cols. ${ }^{25}$ realizaron un estudio desde 2005 a 2016 en el Princess Alexandra Hospital donde obtuvo como resultado que la supervivencia global a los 5 años de los pacientes con carcinoma escamoso metastásico en el cuello sin primario conocido fue de $71,2 \%$. Amsbaugh y cols. ${ }^{13}$ estudiaron 66 pacientes con metástasis cervical de carcinoma escamoso de origen desconocido y obtuvo una tasa de supervivencia global a los 5 años del 69,4\%. Lou y cols. ${ }^{26}$ obtuvieron una supervivencia global a los 5 años del $67,1 \%$. Según tratamiento $71,3 \%$ los que recibieron cirugía y $53,2 \%$ los que no, mientras que Strojan y cols. ${ }^{27}$, en Eslovenia, obtuvieron una tasa de $57 \%$ de supervivencia a los 5 años. En la investigación de Hosni y cols. ${ }^{28}$ solo murieron 10 de 61 pacientes con metástasis cervical de carcinoma de origen desconocido, con una supervivencia global a los 5 años del $83,6 \%$. El estudio de Kamal y cols. ${ }^{29}$ mostró una supervivencia global a los 5 años del $84 \%$ en pacientes con metástasis cervical de primario desconocido tratados con IMRT. Axelsson y cols. ${ }^{30}$ encontraron una supervivencia global a los 5 años de 82\%. Los pacientes HPV positivos tuvieron una supervivencia global del $88 \%$, mientras que los HPV negativos de $61 \%$. Otros autores obtuvieron tasas de supervivencia superior como Shengjin y cols. ${ }^{31}$ en China y Tiong y cols. ${ }^{32}$ en Estados Unidos con $84,5 \%$ y $90 \%$ respectivamente. Solo se encontraron 2 estudios con una tasa de supervivencia menor que fueron el de Al Kadah y cols. ${ }^{21}$ con una supervivencia global a los 5 años de un 30\%, y en el de Weber y cols. ${ }^{17}$ de $13,3 \%$. Cabe destacar que el primero incluía pacientes entre 1991 y 2013, y el segundo de antes de 2001.

Un factor que pudiera estar en relación con este resultado es el hecho de que ha habido a nivel mundial un incremento de los carcinomas de orofaringe p16 positivos, que generalmente se presentan con lesiones ganglionares y primario pequeño $u$ oculto $y$ tienen mucho mejor pronóstico, respuesta al tratamiento y supervivencia que los que no lo $\operatorname{son}^{33,34}$, solo que en nuestro medio aún se está comenzando a realizar esta prueba y en los pacientes objetos de estudio no se conoce el estatus según la infección por HPV, por lo que no podemos descartar que este aumento de la supervivencia podría estar en relación con un mayor número de pacientes con carcinoma de esta etiología. Motz y cols. ${ }^{8}$ realizaron un estudio en el Johns Hopkins Hospital desde 2005 a 2014 donde se les realizó detección de HPV a 84 pacientes con diagnóstico de metástasis de carcinoma escamoso con primario oculto, y obtuvo como resultado que el 90,7\% de los pacientes fueron HPV positivos. Candan y cols. ${ }^{35}$ también obtuvieron como resultado que la mayoría de los pacientes con metástasis cervical de origen desconocido eran HPV positivos y que estos últimos tuvieron mejor supervivencia global a los 5 años. Schroeder y cols. ${ }^{36}$ en un estudio retrospectivo multicentros (Alemania, Italia y España) encontraron que el 89\% de los carcinomas metastásicos cervicales con primario oculto eran HPV positivos y que hubo una tendencia temporal significativa $(\mathrm{p} \leq 0,007)$ al incremento con los años de la prevalencia de la infección viral (del 9\% durante 1998-2004 al 23\% durante 2005-2014). Ross y cols.$^{37}$ compararon la supervivencia global a los 3 años de los pacientes HPV positivos con primario oculto y los HPV positivos de amígdala y obtuvieron como resultado que no hubo gran diferencia entre las tasas, encontrando en ambos una supervivencia global a los 3 años del 91\%, mientras que la supervivencia libre de enfermedad a los 3 años fue del $89 \%$ en los primeros y $85 \%$ en los segundos. 


\section{Análisis de la supervivencia según las diferentes variables}

Nieder y cols. ${ }^{16}$ realizaron una revisión bibliográfica exhaustiva hasta mayo de 2000, donde obtuvieron como resultado que las tasas de supervivencia global a los 5 años oscilan entre $34 \%$ y $63 \%$ con una media del $50 \%$ y que los factores pronósticos determinantes de supervivencia son el estadio N, número de ganglios, extensión extraganglionar y grado histológico. Axelsson y cols. ${ }^{30}$ encontraron como factores determinantes de supervivencia la edad, el estatus según HPV y el estado ganglionar. Según Lou y cols. ${ }^{22}$ el estadio N, metástasis bilateral y presencia de ENE fueron los principales factores para la supervivencia.

Mizuta y cols. ${ }^{24}$ encontraron como factores significativos el estado ganglionar y la extensión extraganglionar. La probabilidad de supervivencia según el estado ganglionar disminuye cuanto más avanzada esté la enfermedad al diagnóstico. En el estudio de Witek y cols. ${ }^{38}$, 36 pacientes con metástasis cervical de carcinoma escamoso de origen desconocido clasificados como N3 tuvieron una supervivencia global a los 5 años del 30\% independientemente del tratamiento recibido.

En los últimos años se ha identificado la extensión extraganglionar como factor adverso independiente del estado ganglionar. En nuestro estudio también resultó determinante de supervivencia. Kharytaniuk y cols. ${ }^{39}$ analizaron la supervivencia de los pacientes con ENE en pacientes $\mathrm{p} 16$ positivos y $\mathrm{p} 16$ negativos con primario oculto y obtuvieron como resultado que la presencia de ENE está asociada a la supervivencia en los pacientes con carcinoma de orofaringe y con primario oculto de acuerdo con el estatus del p16, pues en los pacientes p16 negativo la ENE fue significativamente asociada a una tasa de supervivencia libre de recurrencia peor, mientras que en los p16 positivo no tuvo efecto sobre esta tasa.

\section{Conclusión}

El perfil del paciente con metástasis cervical de carcinoma escamoso de origen desconocido, es un paciente masculino, con una edad de alrededor de 61 años, fumador, con carcinoma pobremente diferenciado, de localización en el nivel II, sin extensión extraganglionar, diagnosticado en estadio N1 y tratados con cirugía y adyuvancia. La supervivencia global a los 5 años de los pacientes con metástasis cervical de primario oculto es relativamente elevada y tiene tendencia a mejorar con el paso de los años. El estado ganglionar y la extensión extraganglionar son variables determinantes de supervivencia, de forma inversamente proporcional.

\section{Bibliografía}

1. Ferlay J, Colombet M, Soerjomataram I, et al. Estimating the global cancer incidence and mortality in 2018: GLOBOCAN sources and methods. Int J Cancer. 2019;144(8):1941-1953.

2. Ferlay J, Ervik M, Lam F, et al. (2018). Global Cancer Observatory: Cancer Today. Lyon, France: International Agency for Research on Cancer. Available from: https://gco.iarc.fr/today, accessed [18/12/2019].

3. Chaturvedi AK, Engels EA, Pfeiffer RM, et al. Human papillomavirus and rising oropharyngeal cancer incidence in the United States. J Clin Oncol. 2011; 29:4294-4301.

4. Rischin D, Young RJ, Fisher R, et al. Prognostic significance of p16INK4A and human papillomavirus in patients with oropharyngeal cancer treated on TROG 02.02 phase III trial. J Clin Oncol. 2010;28:4142-4148.

5. Ang KK, Harris J, Wheeler R, et al. Human papillomavirus and survival of patients with oropharyngeal cancer. N Engl J Med. 2010;363:24-35.

6. Jordan RC, Lingen MW, Perez-Ordonez B, et al. Validation of methods for oropharyngeal cancer HPV status determination in US cooperative group trials. Am J Surg Pathol. 2012;36:945-954.

7. Bochtler T, Löffler H, Krämer A. Diagnosis and management of metastatic neoplasms with unknown primary. Semin Diagn Pathol. 2017;17:147-148.

8. Motz K, Qualliotine JR, Rettig E, Richmon JD, Eisele DW, Fakhry C. Changes in Unknown Primary Squamous Cell Carcinoma of the Head and Neck at Initial Presentation in the Era of Human Papillomavirus. JAMA Otolaryngol Head Neck Surg 2016; 142(3):223-228.

9. Yildirim M, Müller von der Grün J, Winkelmann R, Fokas E, Rödel F, Ackermann H, Rödel C, Balermpas P. Combined p16 and p53 expression in cervical cancer of unknown primary and other prognostic parameters: A single-center analysis. Strahlenther Onkol. 2017;193(4):305-314.

10. Miller FR, Karnad AB, Eng T, Hussey DH, Stan McGuff H, Otto RA. Management of the unknown 
primary carcinoma: long-term follow-up on a negative PET scan and negative panendoscopy. Head Neck. 2008; 30(1):28-34.

11. Kutler DI, Patel SG, Shah JP. The role of neck dissection following definitive chemoradiation. Oncology. 2004;18:993-8.

12. National Comprehensive Cancer Network. Clinical practice guidelines in Oncology-v.1.2018.

13. Amsbaugh MJ, Yusuf M, Gaskins J, et al. Neck dissection for unknown cancer of the head and neck in the era of chemoradiation. Am J Otolaryngol. 2017;38(5):588-92.

14. Xiaomei L, Dianhe L, Na L, Xiaoxia Z. Optimization of radiotherapy for neck carcinoma metastasis from unknown primary sites: a meta-analysis. Oncotarget 2016;7(48): 78736-46.

15. Arosio AD, Pignataro L, Gaini RM, Garavello W. Neck lymph node metastases from unknown primary. Cancer Treat Rev. 2017;53:1-9.

16. Nieder C, Gregoire V, Ang KK. Cervical lymph node metastases from occult squamous cell carcinoma: cut down a tree to get an apple? Int J Radiat Oncol Biol Phys. 2001;3:23.

17. Weber A, Schmoz S, Bootz F. CUP (carcinoma of unknown primary) syndrome in head and neck: clinic, diagnostic, and therapy. Onkologie. 2001;24(1):38-43.

18. Yoo MG, Kim J, Bae S, Ahn SS, Ahn SJ, Koh YW. Detection of clinically occult primary tumours in patients with cervical metastases of unknown primary tumours: comparison of three-dimensional THRIVE MRI, two-dimensional spin-echo MRI, and contrastenhanced CT. Clin Radiol 2018;73(4):410-415.

19. Cianchetti M, Mancuso AA, Amdur RJ, et al. Diagnostic evaluation of squamous cell carcinoma metastatic to cervical lymph nodes from an unknown head and neck primary site. Laryngoscope. 2009;119(12):2348-2354.

20. $\mathrm{PDQ}^{\circledR}$ Adult Treatment Editorial Board. PDQ Metastatic Squamous Neck Cancer With Occult Primary Treatment (Adult). Bethesda, MD: National Cancer Institute. Updated $<02 / 08 / 2018>$. Disponible en: https://www.cancer.gov/types/head-and-neck/hp/ adult/metastatic-squamous-neck-treatment-pdq.

21. Al Kadah B, Papaspyrou G, Linxweiler M, et al. Cancer of unknown primary (CUP) of the head and neck: retrospective analysis of 81 patients. Eur Arch Otorhinolaryngol. 2017;274(6):2557-2566.

22. Dragan AD, Nixon IJ, Guerrero-Urbano MT, Oakley R, Jeannon JP, Simo R. Selective neck dissection as a therapeutic option in management of squamous cell carcinoma of unknown primary. Eur Arch Otorhinolaryngol. 2014; 271(5):1249-1256.

23. Zhou MJ, van Zante A, Lazar AA, et al. Squamous cell carcinoma of unknown primary of the head and neck: Favorable prognostic factors comparable to those in oropharyngeal cancer. Head Neck. 2017; 12:10.
24. Mizuta M, Kitamura M, Tateya I, et al. Unknown primary squamous cell carcinoma of the head and neck: retrospective analysis of 80 cases. Acta Otolaryngol. 2018;1:1-7.

25. Huo M, Panizza B, Bernard A, Porceddu SV. Head and neck squamous cell carcinoma of unknown primary: Outcomes of a pre-defined institutional treatment policy. Oral Oncol. 2018;77:43-48.

26. Lou J, Wang S, Wang K, Chen C, Zhao J, Guo L. Squamous cell carcinoma of cervical lymph nodes from an unknown primary site: The impact of neck dissection. J Cancer Res Ther. 2015;11(2):C161-167.

27. Strojan P, Kokalj M, Zadnik V, et al. Squamous cell carcinoma of unknown primary tumor metastatic to neck nodes: role of elective irradiation. Eur Arch Otorhinolaryngol. 2016;273(12):4561-4569.

28. Hosni A, Dixon PR, Rishi A, et al. Radiotherapy Characteristics and Outcomes for Head and Neck Carcinoma of Unknown Primary vsT1 Base of Tongue Carcinoma. JAMA Otolaryngol Head Neck Surg. 2016;142(12):1208-1215.

29. Kamal M, Mohamed ASR, Fuller CD, et al. Outcomes of patients diagnosed with carcinoma metastatic to the neck from an unknown primary source and treated with intensity-modulated radiation therapy. Cancer 2018;1:10.

30. Axelsson L, Nyman J, Haugen-Cange H, et al. Prognostic factors for head and neck cancer of unknown primary including the impact of human papilloma virus infection. J Otolaryngol Head Neck Surg. 2017;46(1):45.

31. Dou S, Qian W, Ji Q, Wang Z, Zhu G. Tailored multimodality therapy guided by a two-step decision making process for head-and-neck cancer of unknown primary. Oncotarget. 2016;7(26):4009540105.

32. Tiong A, Rischin D, Young RJ, et al. Unilateral radiotherapy treatment for $\mathrm{p} 16 /$ human papillomavirus-positive squamous cell carcinoma of unknown primary in the head and neck. Laryngoscope. 2018; 2:131.

33. Troussier I, Klausner G, Morinière $S$, et al. Advances in the management of cervical lymphadenopathies of unknown primary: advances in diagnostic imaging and surgical modalities and new international staging system. Bull Cancer. 2018;105(2):181-92.

34. Cheol Park G, Roh JL, Cho KJ, et al. 18 F-FDG PET/ CT vs. human papillomavirus, p16 and EpsteinBarr virus detection in cervical metastatic lymph nodes for identifying primary tumors. Int $J$ Cancer. 2017;140(6):1405-1412.

35. Demiroz C, Vainshtein JM, Koukourakis GV, et al. Head and neck squamous cell carcinoma of unknown primary: Neck dissection and radiotherapy or definitive radiotherapy. Head Neck. 2014; 36(11):1589-1595.

36. Schroeder L, Boscolo-Rizzo P, Dal Cin E, et al. 
Human papillomavirus as prognostic marker with rising prevalence in neck squamous cell carcinoma of unknown primary: A retrospective multicentre study. Eur J Cancer. 2017;74:73-81.

37. Ross RB, Koyfman SA, Reddy CA, et al. A matched comparison of human papillomavirus-induced squamous cancer of unknown primary with early oropharynx cancer. Laryngoscope. 2017;10:102.

38. Witek ME, Wieland AM, Chen S, et al. Outcomes for patients with head and neck squamous cell carcinoma presenting with N3 nodal disease. Cancers Head Neck 2017;2:8.

39. Kharytaniuk N, Molony P, Boyle S, et al. Association of Extracapsular Spread With Survival According to Human Papillomavirus Status in Oropharynx Squamous Cell Carcinoma and Carcinoma of Unknown Primary Site. JAMA Otolaryngol Head Neck Surg. 2016;142(7):683-690. 